\title{
Characterization and phylogenetic analysis of new bat astroviruses detected in Gabon, Central Africa
}

\author{
V. ROUGERON ${ }^{1,2}$, E. SUQUET ${ }^{1}$, G. D. MAGANGA ${ }^{1}$, D. JIOLLE ${ }^{1}$, I. M. MOMBO ${ }^{1}$, M. BOURGAREL ${ }^{3}$, P. MOTSCH $^{1}$, \\ C. ARNATHAU ${ }^{2}$, P. DURAND 2 , J. F. DREXLER ${ }^{4}$, C. DROSTEN ${ }^{4}$, F. RENAUD ${ }^{2}$, F. PRUGNOLLE ${ }^{1,2}$, E. M. LEROY $^{1,2}$
}

\begin{abstract}
${ }^{1}$ Centre International de Recherches Médicales de Franceville (CIRMF), BP769, Franceville, Gabon; ${ }^{2}$ Laboratory MIVEGEC, UMR 224-5290 CNRS-IRD-UM, IRD, Montpellier, France; ${ }^{3}$ Centre de Coopération Internationale en Recherche Agronomique Pour le Développement (CIRAD), UPR AGIRS, F-34398 Montpellier, France; ${ }^{4}$ Institute of Virology, University of Bonn Medical Centre, Bonn, Germany
\end{abstract}

Received December 18, 2015; revised March 13, 2016; accepted November 9, 2016

\begin{abstract}
Summary. - Astroviruses are emerging RNA viruses that cause enteropathogenic infections in humans and in other mammals. The identification of astroviruses in a wide range of animals highlights the zoonotic importance of these viruses. Bats can harbor many different viruses, among which some are highly pathogenic for humans (for instance, Nipah, Ebola and SARS coronavirus), and also several astroviruses. As some RNA viruses can be directly transmitted from bats to humans, it is crucial to collect data about their frequency, genetic diversity and phylogenetic characterization. In this study, we report the molecular identification of 44 new astroviruses (with a detection rate of 4.5\%) in 962 apparently healthy bats that belong to five different species and that were captured in different caves in North-East Gabon, Central Africa. Our results show that bat astroviruses form a group that is genetically distinct from astroviruses infecting other mammals. Moreover, these astroviruses showed an important genetic diversity and low host restriction in bat species.
\end{abstract}

Keywords: bat; astroviruses; Gabon; host restriction; genetic diversity

\section{Introduction}

Astroviruses are members of the Astroviridae family and are classified in two genera: Mamastroviruses and Avastroviruses that infect mammals and birds, respectively ( Méndez et al., 2007; De Benedictis et al., 2011). Astroviruses are small, non-enveloped icosahedral viruses of $28-35 \mathrm{~nm}$ in diameter that are characterized by a five- or six-pointed star-like appearance when observed by electron microscopy. These viruses have a positive-strand RNA genome with a $\operatorname{poly}(\mathrm{A})$ tail at the 3 'end, but no cap at the 5 'end. Their genome is about 6,800 to 7,900 nucleotide-long and

E-mail: rougeron.virginie@gmail.com, phone: +33449191869. Abbreviations: $\mathrm{AstV}=$ astrovirus; $\mathrm{CIRMF}=$ Centre International de Recherches Médicales de Franceville; RdRp = RNA-dependent RNA polymerase; RNA = ribonucleic acid; SARS CoV = SARS coronavirus is composed of three ORFs (ORF1a, ORF1b and ORF2) that encode, respectively, the protease, RNA-dependent RNA polymerase (RdRp) and capsid precursor protein. Astroviruses are one of the main causative agents of mild to severe gastroenteritis in humans and other mammals (Moser and Schultz-Cherry 2005). Mamastroviruses have been identified in humans suffering from diarrhea, particularly in infants and young children (De Benedictis et al., 2011). Several reports showed that astroviruses are present worldwide and represent the second most important cause of gastroenteritis in children, after rotaviruses (Herrmann et al., 1991; Matsui et al., 2001; Moser and Schultz-Cherry 2005; De Benedictis et al., 2011). Astroviruses have also been identified in several animal species, such as cats, swine, sheep, minks, cheetahs, sea lions, bottlenose dolphins, red deer, dogs, rodents and bats (Woode and Bridger, 1978; Snodgrass et al., 1979; Gray et al., 1980; Williams, 1980; Tzipori et al., 1981; Bridger et al., 1984; Shirai et al., 1985; Woode et al., 1985; Harbour et al., 1987; Marshall 
et al., 1987; Vieler and Herbst, 1995; Englund et al., 2002; Lukashov and Goudsmit, 2002; Zhu et al., 2009; Blomström et al., 2010; Chu et al., 2010; Reuter et al., 2011; Tse et al., 2011). Recently, new astroviruses (MBL1 and VA1) have been identified in humans with diarrhea (Finkbeiner et al., 2008; 2009a; 2009b). Phylogenetic analyses revealed that these new viruses are genetically divergent from all previously described serotypes and that they are related to rodent, mink or ovine astroviruses (Finkbeiner et al., 2008, 2009a,b). These results suggest that astroviruses can infect several different host species and highlight the risk that animals could be reservoirs of astroviruses pathogenic for humans.

Bats (Chiroptera order) are considered as the second most abundant, diverse and geographically dispersed vertebrate species after rodents (Calisher et al., 2006; Kasso et al., 2013). These animals can harbor more than 80 virus species and are considered to be the reservoir of many emerging virulent viruses, such as lyssaviruses, Nipah and Hendra viruses, SARS coronavirus (CoV), Ebola virus and Marburg virus. Recent studies highlighted the high prevalence rates and important genetic diversity of enteric astroviruses in bats collected in China, Germany and Hungary (Zhu et al., 2009; Chu et al., 2010; Xiao et al., 2011; Drexler et al., 2012; Hu et al., 2014). In this context, studies on the phylogenetic relationships among astroviruses isolated in wild life animals are the first step to better understand the prevalence, genetic diversity and ecology of these viruses.

Here, we report the detection of new bat astroviruses that circulate in five different bat species in Central Africa (Gabon). Our results show that these bat astroviruses are included in the already described bat astrovirus groups. Moreover, our sequence data suggest that bat astroviruses are characterized by an important genetic diversity and by low host restriction among bat species.

\section{Materials and Methods}

Study sites and sample collection. Bats were captured in three caves located in the Belinga mountain complex (Ogooue'-Ivindo province, North-East Gabon): Faucon Cave $\left(1^{\circ} 07 \mathrm{~N} 13^{\circ} 20 \mathrm{E}\right)$, Zadie Cave $\left(0^{\circ} 98 \mathrm{~N} 13^{\circ} 19 \mathrm{E}\right)$ and Batouala Cave $\left(0^{\circ} 82 \mathrm{~N} 13^{\circ} 45 \mathrm{E}\right)$ in July 2009. The distances between the different caves were as follows: $33.7 \mathrm{~km}$ between the Batouala and Zadie caves, $11.7 \mathrm{~km}$ between the Zadie and Faucon caves and $37.7 \mathrm{~km}$ between the Batouala and Faucon caves. Bats were captured with mist nets installed at the cave entrance just before twilight and were individually euthanized. Species identification was done on-site by trained field biologists and their forearm measurements, weight, sex and breeding status were recorded. Samples of different organs (liver, spleen, kidney, lung, heart, intestines, brain and salivary glands) were collected. Samples were frozen and transferred to the Centre International de Recherches Médicales de Franceville (CIRMF) laboratory (Gabon), where they were stored at $-80^{\circ} \mathrm{C}$ until analysis. Molecular analyses were used to test for the presence of astroviruses in 962 bat intestines, collected in the three caves. Captured bats belonged to four insectivorous species (Hipposideros cf. ruber, Hipposideros gigas, Coleura afra and Miniopterus inflatus) and one frugivorous species (Rousettus aegyptiacus).

Ethical approval. Bat capture and sampling were conducted with the permissions of the Wildlife and Hunting Department of the Gabonese Ministry of Water and Forestry ( $\mathrm{N}^{\circ} 003 / \mathrm{MEFE}-\mathrm{PA} /$ SG/DGEF/DCF and No021/MEFE-PA/SG/DGEF/DCF). All the capture events, animal handling, euthanasia and sample transfer across country borders were performed in accordance with the guidelines of the American Society of Mammalogists (http://www. mammalsociety.org/committees/animal-care-and-use) (Gannon and Sikes 2007).

RNA extraction. To save time and money, RNA extracted from pooled intestine samples (five samples/pool) from the same bat species was used. Approximately $100 \mathrm{mg}$ of each intestine sample was pooled and crushed in $500 \mu \mathrm{l}$ of cold phosphate-buffered saline (Biological Diagnostic Supplies Ltd) at 1500 strokes/min in a ballmill tissue grinder (Geno/ Grinder 2000, Spex CertiPrep) for $2 \mathrm{~min}$. One hundred microliters of this suspension was incubated with $300 \mu \mathrm{l}$ of lysis buffer from the EZ1 RNA Tissue Mini Kit (Qiagen, Hilden, DE) (Qiagen) for 10 min and RNA was then extracted according to the manufacturer's recommended procedure and eluted in $100 \mu$ of elution buffer.

$R d R p$ gene amplification by RT-PCR. Each pool was reversetranscribed (RT) and amplified by hemi-nested PCR with primers that target part of the RNA-dependent RNA polymerase (RdRP) gene sequence in the astrovirus genome, adapted from Chu et al. (2008). Specifically, cDNA synthesis and the first round of PCR amplification were performed using the Superscript III One-step RT-PCR kit (Invitrogen) in a $20 \mu$ reaction mixture that contained $5 \mu$ of pooled bat intestine RNA, $2 \mu$ of the primers $(10 \mu \mathrm{mol} / \mathrm{l})$ PoonFwd1a (5'-GARTTYGATTGGRCKCGKTAYGA-3'), PoonFwd1b (5'-GARTTYGATTGGRCKAGGTAYGA-3') and PoonRev (5'-GGYTTKACCCACATNCCRAA -3'), $1 \mu \mathrm{l}$ of $1 \mu \mathrm{g} / \mu \mathrm{l} \mathrm{BSA,} 0.4 \mu \mathrm{l}$ of $50 \mathrm{mmol} / \mathrm{l} \mathrm{MgSO}_{4}$ and $1 \mu \mathrm{l}$ of $1 \mathrm{U}$ Taq polymerase. The RT step $\left(50^{\circ} \mathrm{C}\right.$ for $\left.30 \mathrm{~min}\right)$ was immediately followed by a denaturation step $\left(94^{\circ} \mathrm{C}\right.$ for $\left.3 \mathrm{~min}\right)$ and $45 \mathrm{PCR}$ cycles $\left(94^{\circ} \mathrm{C}\right.$ for $15 \mathrm{sec}, 50^{\circ} \mathrm{C}$ for $30 \mathrm{sec}$ and $68^{\circ} \mathrm{C}$ for $\left.30 \mathrm{sec}\right)$ and finally by an elongation step $\left(68^{\circ} \mathrm{C}\right.$ for 10 $\mathrm{min})$. For the second round of PCR amplification, the Platinum Taq DNA polymerase High Fidelity kit (Invitrogen) was used according to the manufacturer's recommendations. Specifically, $3 \mu$ of the first-round PCR product was mixed with $2 \mu$ lof each of the primers ( $10 \mathrm{mmol} / \mathrm{l}$ ) PoonFwd2a (5'-CGKTAYGATGGKACKATHCC-3'), PoonFwd2b (5'-AGGTAYGATGGKACKATHCC-3') and PoonRev (5'-GGYTTKACCCACATNCCRAA -3'), $5 \mu \mathrm{l}$ of 10x High Fidelity PCR Buffer (Invitrogen), $1 \mu \mathrm{l}$ of dNTPs (10 mM of each dNTP), 2.5 $\mu \mathrm{l}$ of $50 \mathrm{mmol} / 1 \mathrm{MgCl}_{2}$ and $0.2 \mu \mathrm{l}$ of $1 \mathrm{U}$ Platinum Taq High Fidelity ( $50 \mu \mathrm{l}$ of total reaction volume). After an initial denaturation at $94^{\circ} \mathrm{C}$ for $2 \mathrm{~min}, 45$ cycles of PCR amplification were carried out as 
follows: $94^{\circ} \mathrm{C}$ for $15 \mathrm{sec}, 50^{\circ} \mathrm{C}$ for $30 \mathrm{sec}$ and $72^{\circ} \mathrm{C}$ for $30 \mathrm{sec}$ and an elongation step at $72^{\circ} \mathrm{C}$ for $10 \mathrm{~min}$. PCR products (expected amplicon size of $422 \mathrm{bp}$ ) were visualized on $2 \%$ agarose gels stained with GelRed (Biotium). Positive pools were purified with the QIAQuick PCR purification kit (Qiagen) and then sequenced in both directions (SeqLab, Germany). For each positive pool, individual RNAs were extracted from the intestine samples included in that pool, PCR-amplified and positive samples were purified and sent for sequencing, as described above (SeqLab, Germany).

Statistical and phylogenetic analyses. Statistical analyses were carried out using the R software (Hothorn and Everitt, 2015). The partial RdRp sequences were compared with a database of complete sequences of almost all available serotypes in GenBank (Supplementary Table 1) to determine whether these sequences were genetically related to any known astrovirus serotype. Phylogenetic analyses were performed to identify the relation of the astroviruses detected in the bat samples with other astroviruses. Multiple alignments were performed using the CLUSTAL W software, implemented in Geneious Pro v.8.1.14 (Biomatters, Auckland, New Zeland, www. geneious.com) (Larkin et al., 2007; Kearse et al., 2012). Phylogenetic inference was assessed using a Bayesian approach. The substitution model HKY85, the nearest neighbor interchange (NNI) branch swapping and 1000 bootstrap replicates were applied, through MrBayes implemented in Geneious Pro v.8.1.14 (Ronquist and Huelsenbeck, 2003; Kearse et al., 2012).

Nucleotide sequence accession numbers. All 44 sequences were deposited in GenBank Acc. Nos. KU510441 to KU510484.

\section{Results}

Characterization of the astroviruses identified in bat intestines

Among the 193 bat intestine RNA pools (192 pools of five samples and one of two samples), the astrovirus RdRP region could be amplified by RT-PCR analysis in 45 pools, which corresponded to an overall detection rate of $4.57 \%$ (Table 1). PCR amplification and sequence analysis of the individual intestine samples included in the positive pools confirmed that 44 samples were infected by astroviruses. The detection rate varied among the five bat species $(H . c f$. ruber, H. gigas, C. afra, M. inflatus and R. aegyptiacus): $10.32 \%$ for M. inflatus, $8 \%$ for C. afra, $1.23 \%$ for R. aegyptiacus, $4.31 \%$ for H. cf. ruber and $3.10 \%$ for $H$. gigas (Table 1 ). The detection rate was significantly higher in $M$. inflatus than in the other three bat species (R. aegyptiacus, $H$. cf. ruber and $H$. gigas; $P$-values $<0.05)$.

Genetic diversity and phylogenetic analysis of bat astroviruses

To investigate the genetic relationships of the 44 astroviruses isolated in bats with those infecting other animals, phylogenetic analyses were performed using the partial RdRp region obtained by RT-PCR amplification (363 to $378 \mathrm{bp}$ in relation to the considered sequence) (Fig. 1). All bat astroviruses detected in this study clustered with the bat astroviruses of the Mamastrovirus genus, with a bootstrap value of $79 \%$ (Fig. 1). Moreover, our astrovirus sequences shared 44.20 to $50.90 \%$ of nucleotide identity with turkey astroviruses, 47.90 to $59.50 \%$ with human astroviruses and 48.90 to $85.60 \%$ within the bat astrovirus group. Phylogenetic analysis showed that all bat astrovirus sequences were genetically divergent from the sequences of astroviruses infecting humans and other mammals (Fig. 1). Within each of the five bat species under study, the genetic variability was important and the percentage of nucleotide identity for the RdRp sequences detected in each species was $57.10-84.50 \%$ for $H$. gigas, 55.90-97.80\% for H. cf. ruber, 62\% for R. aegyptiacus, $83.60 \%$ for C. afra and $53.70-98.10 \%$ for M. inflatus.

The 44 bat astrovirus sequences obtained in this work belonged to five different bat astrovirus groups, of which four are characterized by host restriction within the bat genus (Fig. 1). Among the $16 \mathrm{RdRp}$ sequences detected in $M$. inflatus intestine samples, 15 grouped within the bat astrovirus (AstV) Group 4 Miniopterus (56.90 to $85.60 \%$ of nucleotides identity) and the other one (09GB424, colored

Table 1. Rate of detection of the astrovirus RdRp gene sequence in intestine samples from 962 bats collected in Gabon

\begin{tabular}{|c|c|c|c|c|c|c|c|c|c|}
\hline \multirow{2}{*}{ Bat family } & \multirow{2}{*}{ Bat species } & \multicolumn{2}{|c|}{ Faucon cave } & \multicolumn{2}{|c|}{ Zadie cave } & \multicolumn{2}{|c|}{ Batouala cave } & \multicolumn{2}{|c|}{ Total } \\
\hline & & $\mathbf{N}$ & n (\%) & $\mathrm{N}$ & n (\%) & $\mathrm{N}$ & n (\%) & $\mathrm{N}$ & n (\%) \\
\hline \multirow{2}{*}{ Hipposideridae } & Hipposideros cf. ruber & 209 & $1(0.48)$ & 72 & $3(4.17)$ & 113 & $13(11.50)$ & 394 & $17(4.31)$ \\
\hline & Hipposideros gigas & 126 & $5(3.97)$ & 47 & $1(2.13)$ & 53 & $1(1.89)$ & 226 & $7(3.10)$ \\
\hline Emballonuridae & Coleura afra & 2 & $0(0)$ & 0 & $1 / 1$ & 23 & $2(8.70)$ & 25 & $2(8.00)$ \\
\hline Miniopteridae & Miniopterus inflatus & 0 & $1 / 1$ & 0 & $1 / 1$ & 155 & $16(10.32)$ & 155 & $16(10.32)$ \\
\hline \multirow[t]{2}{*}{ Pteropodidae } & Rousettus aegyptiacus & 0 & $1 / 1$ & 143 & $1(0.70)$ & 19 & $1(5.26)$ & 162 & $2(1.23)$ \\
\hline & Total & 337 & $6(1.78)$ & 262 & $5(1.91)$ & 363 & $33(9.09)$ & 962 & $44(4.57)$ \\
\hline
\end{tabular}

The family and species of collected bats as well as the number of samples analyzed $(\mathrm{N})$ and the number of positive astrovirus samples $(\mathrm{n}(\%))$ in the three different caves in Gabon are detailed. 


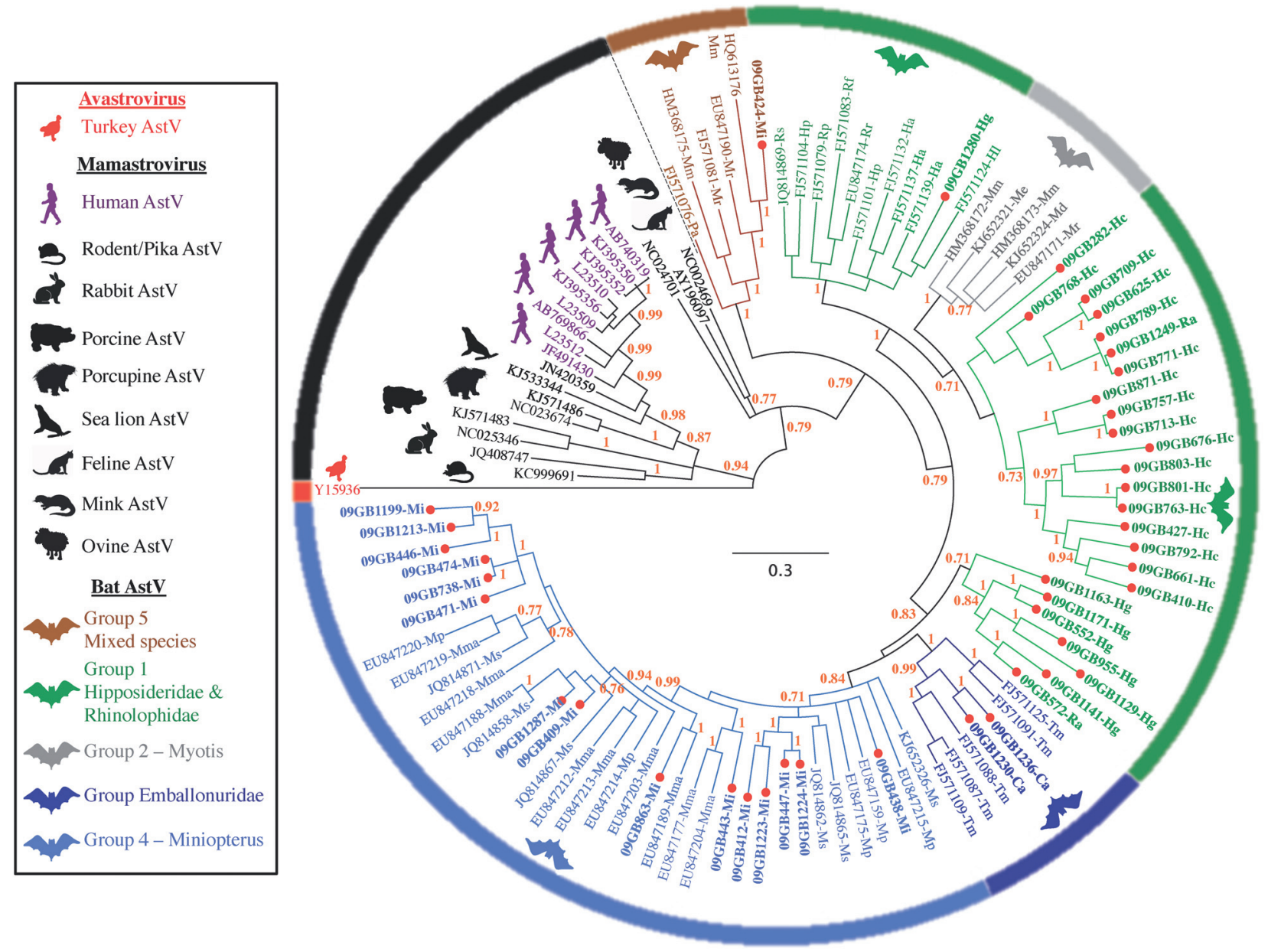

Fig. 1

Phylogenetic tree constructed using a multiple alignment of the partial RdRp gene sequences detected in bat intestine samples collected in three different caves in Gabon

Numbers above the branches indicate the maximum likelihood bootstrap values and only values higher than 70 are indicated. A turkey avastrovirus sequence (Y15936) was used to root the tree. The scale bar indicates the number of substitutions per site (0.3). The 44 astrovirus sequences obtained in this study are indicated by red dots and their names are in bold. The bat species is indicated after the GenBank Acc. No. with the following abbreviations: $\mathrm{Pa}$ (Pipistrellus abramus), $\mathrm{Mr}$ (Myotis ricketti), Me (Myotis emarginatus), Mm (Myotis myotis), Md (Myotis daubentonii), Sk (Scotophilus kuhlii), Ms (Miniopterus schreiberi), Mma (Miniopterus magnater), Mp (Miniopterus pusilus), Rs (Rhinolophus sinicus), Rf (Rhinolophus ferrumequinum), $\mathrm{Rp}($ Rhinolophus pearsonii), $\mathrm{Rr}$ (Rhinolophus rouxii), Tm (Taphozous melanopogon), $\mathrm{Ra}$ (Rousettus aegyptiacus), Mi (Miniopterus inflatus), Hp (Hipposideros pomona), Ha (Hipposideros armiger), $\mathrm{Hl}$ (Hipposideros larvatus), $\mathrm{Hc}$ (Hipposideros cf. ruber), $\mathrm{Hg}$ (Hipposideros gigas) and Ca (Coleura afra). The GenBank Acc. Nos. of the sequences obtained or used in this study are listed in Supplementary Table 1.

in brown in Fig. 1) within the bat AstV Group 5 composed of mixed species (60 to $73.30 \%$ of nucleotide identity). The two RdRp sequences detected in C. afra (09GB1236 and 09GB1230, colored in dark blue in Fig. 1) were included in a group composed of astroviruses detected in one bat species (Taphozous melanopogon) that is part of the Emballonuridae family, which includes the Coleura genus (68.90 to $74.30 \%$ of nucleotides identity). We thus named this group 'Embellonuridae'. All H. cf. ruber RdRp sequences $(\mathrm{n}=17)$ clustered together in one group with a bootstrap of $73 \%$. When comparing these $H$. $c f$. ruber RdRp sequences with the closest ones obtained from the literature, the nucleotide identity varied from 66.60 to $71 \%$. Six of the seven $H$. gigas RdRp sequences formed a sister cluster with the AstV Groups Emballonuridae and Miniopterus with a bootstrap value of $71 \%$, whereas the last one (09GB1280) grouped within the bat AstV Group 1 Hipposideridae and Rhinolophidae. These H. gigas RdRp sequences shared with the closest published sequences between 71 and $74.80 \%$ of nucleotide identity. The two RdRp sequences detected in R. aegyptiacus (09GB572 and 09GB1249) grouped within the two new groups composed of astroviruses detected in $H$. cf. ruber or $H$. gigas 
Table 2. Detection of the astrovirus RdRp gene sequence in the 962 bats in relation to the cave where they were captured

\begin{tabular}{llll}
\hline & Faucon cave & Zadie cave & Batouala cave \\
\hline Faucon cave & $5 / 262(1.61)$ & $P=0.909$ & $\boldsymbol{P}=\mathbf{0 . 0 0 0 4}$ \\
\hline Zadie cave & & $6 / 337(1.78)$ & $\boldsymbol{P}=\mathbf{0 . 0 0 0 0 6}$ \\
\hline Batouala cave & & & $33 / 3639.09)$ \\
\hline
\end{tabular}

The number of analyzed samples and the number of positive samples in each cave are indicated $(\mathrm{n} / \mathrm{N}(\%))$. The chi-square test was used to compare the detection rates in the three caves. Significant $P$-values $(P)$ are indicated in bold.

(58.90 to $68.20 \%$ of nucleotide identity). As the astrovirus RdRp sequences detected in $H$. gigas and $H$. c ff. ruber were from the same virus family, all these sequences clustered within the AstV Group 1 Hipposideridae and Rhinolophidae (Fig. 1). These results confirm that bat astroviruses are characterized by an important genetic diversity, as previously described (Chu et al., 2008; Zhu et al., 2009; Xiao et al., 2011; Drexler et al., 2012; Hu et al., 2014). Moreover, astroviruses do not seem to show host restriction within bat species.

\section{Bat astroviruses do not show geographic structuration}

When considering the astrovirus distribution in the three caves, no significant bat astrovirus phylogenetic clustering within each cave was observed (results not shown in the phylogeny, Fig. 1). Indeed, the viruses isolated in a given bat species could have been from animals collected in any of the three caves with no significant difference (Table 2). Conversely, the detection rate of astrovirus-positive samples was different in the three caves. Specifically, the astrovirus detection rate in the Batouala cave, where all five bat species were captured, was significantly higher $(9.09 \%, P$-value $<0.05)$ than in the Faucon and Zadie caves, where no $M$. inflatus bats were collected $(1.78 \%$ and $1.61 \%$, respectively) (Table 2 ).

\section{Discussion}

To improve the current knowledge on bat astrovirus diversity and epidemiology, we investigated five African bat species (H. cf. ruber, H. gigas, C. afra, M. inflatus and $R$. aegyptiacus) collected in Gabon. We found that all five bat species carried astroviruses, with variable detection rates (from $10.32 \%$ to $1.23 \%$ ). Moreover, insectivorous ( $M$. inflatus, H. cf. ruber, H. gigas and C. afra) as well as frugivorous ( $R$. aegyptiacus) bats seem to harbor astroviruses without any host restriction. Our astrovirus detection rate was lower than what reported in previous studies on bats collected in China (Zhu et al., 2009; Chu et al., 2010; Xiao et al., 2011;
Hu et al., 2014). However, in agreement with these studies (Zhu et al., 2009; Xiao et al., 2011), the highest astrovirus detection rate was in bats of the genus Miniopterus.

The phylogenetic analysis showed the existence of a distinct phylogenetic sister-group of astroviruses circulating in bats, in agreement with previous works (Zhu et al., 2009; Xiao et al., 2011). This bat AstV group is not included within the currently known diversity of astroviruses circulating in humans (or in other mammals) and forms a divergent group. Our data show that all astrovirus sequences detected in $H$. cf. ruber and six of the seven sequences identified in H. gigas intestine samples formed two distinct groups within the AstV Group 1. These two new phylogenetic groups need to be validated as independent from, or still included in the AstV Group 1 by whole genome sequencing. When considering the geographic structuration, no significant phylogenetic clustering of bat astroviruses in the three different caves, where samples were collected, was observed. Indeed, viruses isolated from one bat species could have been from animals captured in any of the three caves. This could be explained by the bat lifestyle, for instance the migration of hundreds of individuals at the same time of the day, thus allowing potential contacts between individuals.

Currently, the species tropism of bat astroviruses is still debated. Some authors suggested that astrovirus transmission and infection have strict host tropism (Baxendale and Mebatsion 2004), whereas others found that bat astroviruses are phylogenetically related to different mammal species (such as minks, ovine and humans) and, therefore, do not show strict host specificity (Xiao et al., 2011). In the present study, among the 44 bat astrovirus sequences we detected, 43 sequences clustered within four bat AstV groups that are characterized by host restriction within the bat genus. This suggests a low degree of host species restriction within the bat species and high capacity of adaption to different bat species. This is in line with the occurrence of multiple cross-species transmission events among different bat species, and probably with other mammal species, that is facilitated by the bat lifestyle (i.e., several bat species living in the same cave), the astrovirus tropism and the oral-fecal transmission of these viruses. On the other hand, one astrovirus detected in M. inflatus (09GB424) clustered in the bat AstV Group 5 that includes astroviruses from bat species from different families and is phylogenetically distinct from the other bat AstV groups. This bat AstV group is the only one composed of astroviruses infecting different bat genera and therefore, it could represent the bridge group of bat astroviruses that can adapt to and infect different bat species. Whole genome sequencing analysis of recombination events in bat and other animals' astroviruses should allow confirming this hypothesis.

Genetically, the 44 bat astrovirus sequences (detected in five different bat species) were remarkably diverse (nucleotide sequence similarity between $53.70 \%$ and $98.10 \%$ ). 
The high genetic diversity of astroviruses (De Benedictis et al., 2011) associated with the wide variety of host species infected by these viruses suggest and confirm (i) their efficiency in cross-species transmission, (ii) their great adaptability to new environments (hosts) and (iii) their potential zoonotic capability (Lukashov and Goudsmit, 2002). It seems that these viruses evolved in bats for a very long time and therefore, bats could be considered as potential reservoirs of astroviruses.

Finally, unlike Ebola virus, Marburg virus, SARS CoV, Hendra virus or Nipah virus, for which transfers from bats to humans have been documented (Calisher et al., 2006; Han et al., 2015; Marí Saéz et al., 2015), the bat astrovirus group seems to be highly independent from astroviruses that infect other mammalian species, including humans. Indeed, none of the bat RdRp sequences obtained in this and all previous studies belongs to the group of astroviruses that can infect other mammalian species. On the basis of this observation and of the high genetic diversity of bat astroviruses, we hypothesize that these astroviruses are characterized by a very specific host invasion pathway that limits cross-species transfers. This needs to be tested, particularly because the main astrovirus cell receptor has not yet been identified.

In conclusion, this study brings new knowledge on bat astrovirus genetic diversity and epidemiology in Gabon. We molecularly characterized 44 new RdRp sequences from astroviruses that circulate in five different bats species, collected in three caves in North-East Gabon, Central Africa. Our results show that these astroviruses are characterized by a high genetic diversity and low bat species-specificity. Today, emerging and reemerging infectious diseases that affect wild and domestic animals as well as human populations constitute a major public health issue (Wolfe, 2005). In this context, more effort should be focused on fighting newly emerging viruses. One way is to evaluate the diversity of astroviruses circulating in wildlife worldwide, because previous studies focused on restricted geographical areas (Chu et al., 2008; Xiao et al., 2011; Drexler et al., 2012; Hu et al., 2014). Another way is to study recombination events between astrovirus genomes isolated in different host species. Indeed, a major question is whether an astrovirus that naturally circulates in bats (or in another animal species) could recombine with a human variant and create a new variant that can infect another animal population, including humans (De Benedictis et al., 2011).

Acknowledgements. We thank the Gabonese Government and Total for their financial support. The authors acknowledge E. Andermarcher for English corrections and editing of the manuscript.

Supplementary information is available in the online version of the paper.

\section{References}

Baxendale W, Teshome M (2004): The Isolation and characterisation of astroviruses from chickens. Avian Pathol. J. W.V.P.A 33, 364-370. https://doi.org/10.1080/0307945042000220426

Blomström AL, Frederik W, Hammer AS, Belák S, Berg M (2010): Detection of a Novel Astrovirus in Brain Tissue of Mink Suffering from Shaking Mink Syndrome by Use of Viral Metagenomics. J. Clin. Microbiol. 48, 4392-4396. https:// doi.org/10.1128/JCM.01040-10

Bridger JC, Hall GA, Brown JF (1984): Characterization of a Calici-like Virus (Newbury Agent) Found in Association with Astrovirus in Bovine Diarrhea. Infect. Immun. 43, 133-138.

Calisher CH, Childs JE, Field HE, Holmes VK, Schountz T (2006): Bats: Important Reservoir Hosts of Emerging Viruses. Clin. Microbiol. Rev. 19, 531-545. https://doi. org/10.1128/CMR.00017-06

Chu DKW, Chin AWH, Smith GJ, ChanK-H, Guan Y, Malik Peiris JS, Poon LLM (2010): Detection of Novel Astroviruses in Urban Brown Rats and Previously Known Astroviruses in Humans. J. Gen. Virol. 91, 2457-2462. https://doi. org/10.1099/vir.0.022764-0

Chu DKW, Poon LLM, Guan Y, Peiris JSM (2008): Novel Astroviruses in Insectivorous Bats. J. Virol. 82, 9107-9114. https://doi.org/10.1128/JVI.00857-08

De Benedictis P, Schultz-Cherry S, Burnham A, Cattoli G (2011): Astrovirus Infections in Humans and Animals - Molecular Biology, Genetic Diversity, and Interspecies Transmissions. Infect. Genet. Evol. 11, 1529-1544. https://doi. org/10.1016/j.meegid.2011.07.024

Drexler JF, Corman VM, Müller MA, Maganga GD, Vallo P, Binger T, Gloza-Rausch F, et al. (2012): Bats Host Major Mammalian Paramyxoviruses. Nat. Commun. 3, 796. https:// doi.org/10.1038/ncomms1796

Englund L, Chriél M, Dietz HH, Hedlund KO (2002): Astrovirus Epidemiologically Linked to Pre-Weaning Diarrhoea in Mink. Vet. Microbiol. 85, 1-11. https://doi.org/10.1016/ S0378-1135(01)00472-2

Finkbeiner SR, Kirkwood CD, Wang D (2008): Complete Genome Sequence of a Highly Divergent Astrovirus Isolated from a Child with Acute Diarrhea. Virol. J. 5, 117. https://doi. org/10.1186/1743-422X-5-117

Finkbeiner SR, Le BM, Holtz LR, Storch GA, Wang D (2009): Detection of Newly Described Astrovirus MLB1 in Stool Samples from Children. Emerg. Infect. Dis. 15, 441-444. https://doi.org/10.3201/1503.081213

Gannon WL, Sikes RS (2007): Guidelines of the American Society of Mammalogists for the Use of Wild Mammals in Research. J. Mammal. 88, 809-823. https://doi.org/10.1644/06MAMM-F-185R1.1

Gray EW, Angus KW, Snodgrass DR (1980): Ultrastructure of the Small Intestine in Astrovirus-Infected Lambs. J. Gen. Virol. 49, 71-82. https://doi.org/10.1099/0022-1317-49-1-71

Han HJ, Wen HL, Zhou CM, Chen FF, Luo LM, Liu JW, Yu XJ (2015): "Bats as Reservoirs of Severe Emerging Infectious Diseases." Virus Res. 205, 1-6. https://doi.org/10.1016/j. virusres.2015.05.006 
Harbour DA, Ashley CR, Williams PD, Gruffydd-Jones TJ (1987): Natural and Experimental Astrovirus Infection of Cats. Vet. Rec. 120, 555-557. https://doi.org/10.1136/ vr.120.23.555

Herrmann JE, Taylor DN, Echeverria P, Blacklow NR (1991): Astroviruses as a Cause of Gastroenteritis in Children. N. Engl. J. Med. 324, 1757-1760. https://doi.org/10.1056/ NEJM199106203242501

Hothorn T, Everitt BS "A Handbook of Statistical Analyses Using R. Third Edition - Google Books." 2015. Accessed October 26.

Hu B, Chmura AA, Li J, Zhu G, Desmond JS, Zhang Y, Zhang W, Epstein JH, Daszak P, Shi Z (2014): Detection of Diverse Novel Astroviruses from Small Mammals in China. J. Gen. Virol. 95, 2442-2449. https://doi.org/10.1099/ vir.0.067686-0

Kasso M, Balakrishnan M (2013): Ecological and Economic Importance of Bats (Order Chiroptera), Ecological and Economic Importance of Bats (Order Chiroptera). International Scholarly Research Notices (November): e187415.

Kearse M, Moir R, Wilson A, Stones-Havas S, Cheung S, Sturrock S, Buxton S et al. (2012): Geneious Basic: An Integrated and Extendable Desktop Software Platform for the Organization and Analysis of Sequence Data. Bioinformatics 28, 1647-1649. https://doi.org/10.1093/bioinformatics/ bts199

Larkin MA, Blackshields G, Brown NP, Chenna R, McGettigan PA, McWilliam H, Valentin F et al. (2007): Clustal W and Clustal X Version 2.0. Bioinformatics 23, 2947-2948. https://doi.org/10.1093/bioinformatics/btm404

Lukashov VV, Goudsmit J (2002): Evolutionary Relationships among Astroviridae. J. Gen. Virol. 83, 1397-1405. https:// doi.org/10.1099/0022-1317-83-6-1397

Marí S, Almudena S, Weiss S, Nowak K, Lapeyre V, Zimmermann F, Düx A, Kühl HS et al. (2015): Investigating the Zoonotic Origin of the West African Ebola Epidemic. EMBO Mol. Med. 7, 17-23. https://doi.org/10.15252/ emmm.201404792

Marshall JA, Kennett ML, Rodger SM, Studdert MJ, Thompson WL, Gust ID (1987): Virus and Virus-like Particles in the Faeces of Cats with and without Diarrhoea. Aust. Vet. J. 64, 100-105. https://doi.org/10.1111/j.1751-0813.1987. tb09638.x

Matsui SM, Kiang D, Ginzton N, Chew T, Geigenmüller-Gnirke U (2001): Molecular Biology of Astroviruses: Selected Highlights. Novartis Foundation Symposium 238, 219-33; discussion 233-36. https://doi.org/10.1002/0470846534. $\underline{\operatorname{ch} 13}$

Méndez E, Aguirre-Crespo G, Zavala G, Arias CF (2007): Association of the Astrovirus Structural Protein VP90 with
Membranes Plays a Role in Virus Morphogenesis. J. Virol. 81, 10649-10658. https://doi.org/10.1128/JVI.00785-07 Moser LA, Schultz-Cherry S (2005): Pathogenesis of Astrovirus Infection. Viral Immunol. 18, 4-10. https://doi.org/10.1089/ vim.2005.18.4

Reuter G, Pankovics P, Boros A (2011): Identification of a Novel Astrovirus in a Domestic Pig in Hungary. Arch. Virol. 156, 125-128. https://doi.org/10.1007/s00705-010-0827-5

Ronquist F, Huelsenbeck JP (2003): MrBayes 3: Bayesian Phylogenetic Inference under Mixed Models. Bioinformatics 19, 15721574. https://doi.org/10.1093/bioinformatics/btg180

Shirai J, Shimizu M, Fukusho A (1985): Coronavirus-, Calicivirus-, and Astrovirus-like Particles Associated with Acute Porcine Gastroenteritis. Nihon Juigaku Zasshi. The Japanese J. Vet. Sci. 47, 1023-1026.

Snodgrass DR, Angus KW, Gray EW, Menzies JD, Paul G (1979): Pathogenesis of Diarrhoea Caused by Astrovirus Infections in Lambs. Arch. Virol. 60, 217-226. https://doi. org/10.1007/BF01317493

Tse H, Chan WM, Tsoi HW, Fan RYY, Lau CCY, Lau SKP, Woo PCY, Yuen KY (2011): Rediscovery and Genomic Characterization of Bovine Astroviruses. J. Gen. Virol. 92, 1888-1898. https://doi.org/10.1099/vir.0.030817-0

Tzipori S, Menzies JD, and Gray EW (1981): Detection of Astrovirus in the Faeces of Red Deer. Vet. Rec. 108, 286. https://doi. org/10.1136/vr.108.13.286

Vieler E, Herbst W (1995): [Electron microscopic demonstration of viruses in feces of dogs with diarrhea]." Tierärztliche Praxis 23, 66-69.

Williams FP, Jr. (1980): Astrovirus-Like, Coronavirus-Like, and Parvovirus-like Particles Detected in the Diarrheal Stools of Beagle Pups. Arch. Virol. 66, 215-226. https://doi. org/10.1007/BF01314735

Wolfe N D (2005): Bushmeat Hunting, Deforestation, and Prediction of Zoonotic Disease Emergence. Emerg. Infect. Dis. 11, 1822-1827. https://doi.org/10.3201/eid1112.040789

Woode GN, Bridger JC (1978): Isolation of Small Viruses Resembling Astroviruses and Caliciviruses from Acute Enteritis of Calves. J. Med. Microbiol. 11, 441-452. https://doi. org/10.1099/00222615-11-4-441

Woode GN, Gourley NE, Pohlenz JF, Liebler EM, Mathews SL, Hutchinson MP (1985): Serotypes of Bovine Astrovirus. J. Clin. Microbiol. 22, 668-670.

Xiao J, Li J, Hu G, Chen Z, Wu Y, Chen Y, Chen Z et al. (2011): Isolation and Phylogenetic Characterization of Bat Astroviruses in Southern China. Arch. Virol. 156, 1415-1423. https://doi.org/10.1007/s00705-011-1011-2

Zhu HC, Chu DKW, Liu W, Dong BQ, Zhang SY, Zhang JX, Li LF et al. (2009): Detection of Diverse Astroviruses from Bats in China. J. Gen. Virol. 90, 883-887. https://doi.org/10.1099/ vir.0.007732-0 